\title{
Privacy-Preserving Payload-Based Correlation for Accurate Malicious Traffic Detection
}

\author{
Janak J. Parekh, Ke Wang, Salvatore J. Stolfo \\ Department of Computer Science, Columbia University \\ 500 W. 120th St., 450 Computer Science Building \\ New York, NY 10027 \\ \{janak,kewang,sal\}@cs.columbia.edu
}

\begin{abstract}
With the increased use of botnets and other techniques to obfuscate attackers' command-and-control centers, Distributed Intrusion Detection Systems (DIDS) that focus on attack source IP addresses or other header information can only portray a limited view of distributed scans and attacks. Packet payload sharing techniques hold far more promise, as they can convey exploit vectors and/or malcode used upon successful exploit of a target system, irrespective of obfuscated source addresses. However, payload sharing has had minimal success due to regulatory or business-based privacy concerns of transmitting raw or even sanitized payloads. The currently accepted form of content exchange has been limited to the exchange of known-suspicious content, e.g., packets captured by honeypots; however, signature generation assumes that each site receives enough traffic in order to correlate a meaningful set of payloads from which common content can be derived, and places fundamental and computationally stressful requirements on signature generators that may miss particularly stealthy or carefully-crafted polymorphic malcode.

Instead, we propose a new approach to enable the sharing of suspicious payloads via privacy-preserving technologies. We detail the work we have done with two example payload anomaly detectors, PAYL and Anagram, to support generalized payload correlation and signature generation without releasing identifiable payload data and without relying on single-site signature generation. We present preliminary results of our approaches and suggest how such deployments may practically be used for not only cross-site, but also cross-domain alert sharing and its implications for profiling threats.
\end{abstract}

\section{Keywords}

Distributed intrusion detection, anomaly detection, privacy preservation, payload correlation, signature generation

\section{INTRODUCTION}

A Distributed Intrusion Detection/Collaborative Intrusion Detection System (DIDS/CIDS) is one that employs multiple Network

Permission to make digital or hard copies of all or part of this work for personal or classroom use is granted without fee provided that copies are not made or distributed for profit or commercial advantage and that copies bear this notice and the full citation on the first page. To copy otherwise, to republish, to post on servers or to redistribute to lists, requires prior specific permission and/or a fee.

SIGCOMM'06 Workshops September 11-15, 2006, Pisa, Italy.

Copyright 2006 ACM 1-59593-417-0/06/0009 ...\$5.00.
Intrusion Detection and/or Host Intrusion Detection sensors (NIDS/ HIDS), often across multiple local area networks, and correlates resulting alerts to get a broader picture of Internet-based threats. Most existing approaches (see section 5) share header-based alert data, e.g., source IPs, destination ports, and aggregate statistics; the goal of correlating such features across multiple sites is to detect common sources of attack, especially as they are performing initial scans to build hitlists in a future attack. Not only can these lists be used in building fast-propagating worms [36], they can also be used for targeted attacks, e.g., an attacker looking to exploit a critical infrastructure industry that may use common services, such as the financial services industry.

However, the advent of botnets [7, 10, 33] and other forms of indirection have made it far more difficult to discover the true attack source, instead of bot machines which play a small role in the actual process. While firewalling can be employed against common IPs [49], there is no guarantee an attacker will not scan using one network and attack using another, thereby defeating proactive attempts.

Instead, one can approach the problem from the perspective of detecting the actual exploit used in the attack attempt: in an increasingly monocultured-software world, specific vulnerabilities are common to a large pool of applications [27]. We can leverage the commonality of such attack approaches (and, in particular, the "invariant substrings" as defined in [30] and others) to identify and protect against such attacks even if target machines are unpatched and remain vulnerable. This is particularly advantageous for zeroday worm detection, when common attack vectors may present themselves across many sites in short timeframes, i.e. correlation of common alerts across space, and for stealthy scanning for long time periods, i.e. correlation of common alerts across time. In either case, correlating alerts among collaborating sites requires careful design for accuracy and efficiency.

Of course, exploit-specific vulnerability detection has its challenges: in particular, a reliance on payload detection and correlation is necessary. It is impractical to assume that organizations can exchange raw traffic streams; there is far too much data of a potentially sensitive nature. Even if exchanged material is confined merely to suspicious payloads as classified by an anomaly detector, organizations may fear that some legitimate and/or sensitive traffic may be misclassified and exchanged to other, possibly competing institutions. Instead, techniques are required to exchange privacypreserving alerts that make it impossible for other entities to determine the actual content of the underlying traffic, yet at the same time exchanging information that can effectively be correlated. We propose that this is not only possible, but practical and broadly applicable, and propose a collection of techniques to do so.

This paper is organized as follows. Section 2 briefly discusses 
the concepts of payload anomaly detection, and introduces two detectors developed by our group-PAYL and Anagram - as a representative class of local detectors. Section 3 then introduces the privacy-preserving correlation techniques at the heart of this paper. Section 4 shows some early results based on the techniques described in section 3 . We discuss related work in section 5 , briefly look at future possibilities in section 6 and conclude in section 7 .

\section{PAYLOAD ANOMALY DETECTION}

In order to better motivate the correlation techniques described in this paper, we first describe two payload anomaly sensors developed at Columbia: PAYL, which implements anomaly detection based on frequency-based 1-gram modeling, and Anagram, which uses binary-based mixtures of higher order $n$-gram modeling $(n>1)$. Both sensors train on normal unencrypted content flows and employ service-specific models to test for suspicious traffic. ${ }^{1}$ Alerts are generated on traffic sufficiently deviant from normal; it is these alerts that we wish to share with other sites to resolve false positives from true zero-day attacks. The reader is encouraged to refer to $[46,44,45]$ for detailed descriptions of the aforementioned sensors.

It is also important to note that we do not intend to address all possible payload detection techniques here. Consequently, the techniques described in section 3 may be usable with other (possibly host-based and/or misuse) sensors.

\subsection{PAYL: 1-gram frequency modeling}

PAYL's models are 1-gram byte frequency distributions conditioned on packet length; tested traffic is classified as normal or malicious by computing the Mahalanobis distance between the distribution of the candidate packets and the frequency model. A larger distance means bigger deviation from the model and a more abnormal packet; thresholding differentiates normal from malicious traffic.

A raw PAYL alert typically contains metadata, including the source and target IP/port pair, payload length, and score (distance from model). Additionally, the suspicious packet may be included in its alert. While the payloads can be shared, they significantly increase alert sizes and run into privacy issues, especially for misclassified traffic, i.e. false positives. While PAYL's false positive rates have been determined to be very low [44], the notion of transmitting any raw payload inhibits collaboration among defensive sites.

\subsection{Anagram: n-gram binary modeling}

Anagram uses an alternative approach to anomaly detection via binary-based high order n-gram modeling. Compared to 1-gram, higher order n-grams are better at modeling sequential content information in packets, and thus it is capable of detecting significant anomalous byte sequences and their location within a packet. To avoid significant memory overhead associated with n-gram frequency distributions, only a binary (yes/no) statistic is kept for each possible gram. Scoring is accomplished by counting the percentage of not-seen-before (i.e. unusual) n-grams out of the total n-grams in the packet, and thresholding is again applied to differentiate traffic.

Surprisingly, analysis shows [45] that binary-based modeling produces extremely good results; it turns out the additional data representation of frequency-based modeling is less advantageous when the space of potential grams grows significantly (e.g., the likelihood of having significant frequency information for distinct 5-grams, or $256^{5}$ grams, is significantly smaller than for the 256 distinct

\footnotetext{
${ }^{1}$ Anagram utilizes other information and is semi-supervised.
}

1-gram), and the representational power of higher-order n-grams effectively offsets the loss of frequency information.

The structure of a raw Anagram alert is similar to that of a raw PAYL alert.

\subsubsection{Bloom filters}

Even though binary-based modeling significantly reduces space overhead, there is still a significant number of possible n-grams as $n$ increases, and a typical hash set structure uses at least 4 bytes per entry. Since only the binary set property is needed, we can use a more efficient, bit-based representation to store the model, reducing data requirements by an order of magnitude. A Bloom filter [5] is one such structure; it is represented as a bit array of $n$ bits, where any individual bit $i$ is set if the hash of an input value, $\bmod n$, is $i$.

A Bloom filter contains no false negatives, but may contain false positives if collisions occur; the false positive rate can be optimized by changing the size of the bit array to avoid saturation, as well as using multiple hash functions (and requiring all of them to be set for an item to be verified as present in the Bloom filter). Operations on a Bloom filter are also $O(1)$, keeping computational overhead low. Finally, a Bloom filter has interesting privacy-preserving properties; we explore these in the next section.

\section{CORRELATION TECHNIQUES}

In this section, we describe several techniques (both raw and privacy-preserving) to support content-based alert correlation. First, however, we develop several metrics as to how we can best compare these techniques.

\subsection{Evaluating correlation techniques}

The techniques described in this paper essentially trade off the amount of information contained versus the privacy maintained. On one extreme, we can consider the idea of transmitting the raw packets that generated alerts; while this enables any correlation technique, we consider it infeasible because of the sheer amount of data and the fact it is not privacy-preserving. On the other end of the spectrum, we can consider privately-encrypted packet content: unless the key is shared, it essentially appears as noise to peersbut this requires all or no trust. The techniques in this paper fall somewhere in between, and we characterize their relative merits from two perspectives: our ability to correlate data given a transformed version of packets and the amount of privacy that is gained using different privacy-preserving transformations of packet content.

Correlation ability. The fundamental question, given any technique, is whether it is possible to correlate alerts with low false positive and low false negative rates. Given raw packets that generate an alert, there are several well-defined algorithms that aim to accomplish this task. We consider the longest common subsequence, or LCSeq, as an appropriate baseline, as it is able to find any non-semantic commonality in the candidate packets, and discuss it below. Other approaches, including semantic matching, are discussed briefly in section 5, and are considered outside the scope of this paper, which focuses on correlation amongst pure network sensors, i.e. no host-specific information.

Given a technique, and a collection of alerts, we can then compute a similarity score distribution as each pair of alerts is tested (see section 4.1). This score distribution then becomes a useful metric for comparing correlation ability. If we consider LCSeq as a useful baseline, for instance, we can measure the deviation of other techniques from LCSeq as a comparative measure of how other techniques correlate alerts. Ideally, a network sensor would be able to use a privacy-enabled technique and get similar results, signify- 
ing an increase in the privacy preservation while maintaining the ability to determine common threats and exploits.

Privacy gain. We characterize the baseline as having no privacy as raw packets are exchanged, and having total privacy with encrypted content without the corresponding key (noise). To characterize intermediate approaches, we utilize a probabilistic model: given a representation of the encoded payload, what is the likelihood that a curious peer would be able to reconstruct the original, possibly sensitive data? For most of the approaches listed, we can estimate this probability by determining the number of original payloads that could be represented by the encoded alert; the resulting measurements are discussed in section 4.4 .

Correlation speed. Finally, one remaining important characteristic is the ability to correlate quickly, especially if many sites are involved with many alerts being generated and exchanged. This "speed" metric is reflected in two aspects: the resulting alert size after a transformation is applied, and the computation overhead necessary to transform the original alert. As with the previous cases, we consider raw packets the baseline: it is the largest unencrypted alert encoding (up to 1500 bytes, i.e. bounded by packet size, per alert) and LCSeq is amongst the slowest correlation mechanisms (up to polynomial-time with respect to buffer size).

\subsection{Alert correlation}

We correlate content alerts using three main approaches: raw packet alert correlation, frequency-based alert correlation, and ngram alert correlation. Techniques for other alerts (e.g., IP alerts) are considered outside the scope of this paper.

\subsubsection{Baseline: Raw payload correlation}

As previously discussed, we choose raw packet alert correlation as a baseline technique: it contains the most complete original information.

SE: String Equality. This is the simplest and most intuitive correlation approach. Two alerts are deemed similar to each other only if they have identical content. This metric is very strict and does minimize false positives, but has no tolerance for any variationfragmentation, polymorphism, obfuscation, etc. Equality is memory and computationally efficient (linear time).

LCS: Longest Common Substring. LCS is one of the classic string comparison techniques; it is less deterministic than SE, and is not susceptible to fragmentation. The longer the string that LCS computes, the greater the confidence that the compared alerts are similar. While it allows minor payload manipulation, multiple changes often cause a short LCS, reducing confidence in its correlation ability. LCS is reasonably fast; a suffix-tree implementation is linear-time, but at the cost of having to store a suffix tree per alert (or $O\left(n^{2}\right)$ for a naive but memory-efficient algorithm).

LCSeq: Longest Common Subsequence. LCSeq can be considered a generalization of LCS; instead of finding a single contiguous matching block, LCSeq allows non-matching characters to be interposed. This enables detection despite a variety of payload manipulation operations, including insertion and reordering, and potentially polymorphism. Like LCS, the length of a LCSeq is an indication of similarity. Its main shortcoming is its computation overhead; at best, sparse dynamic programming can achieve, on average, $O(n \lg n)$ complexity (and can range to $O\left(n^{2} \lg n\right)$ worstcase).

ED: Edit Distance. Edit distance, also known as Levenshtein distance, is another commonly-used approach to compare string similarity. It computes the smallest number of insertions, deletions, and substitutions required to change one string into another. In general, it has similar properties as LCSeq.

\subsubsection{Frequency-modeled 1-gram alert correlation}

Having discussed different techniques for raw payload comparison and correlation, we now describe our first alert transformation: frequency modeling. As our work on PAYL demonstrates [46], 1gram frequency models are a good indicator of the nature of packet content. We can leverage this technique and use frequency distributions as alerts, either with the corresponding normalized frequency counts or with an approximation of this information.

Frequency Distribution. A packet payload can be represented by its byte frequency distribution, making it nearly impossible to reconstruct the actual payload except in degenerate cases- the byte distribution contains byte values but no sequential information. Given two packets with their respective distributions, we can apply standard distance metrics to determine similarity; Manhattan distance is efficient $(O(n)$ in length of the alert) yet produces a good approximation of the actual distance. Frequency-based alerts are comparatively sized compared to packets; a floating-precision frequency distribution takes $1 \mathrm{~KB}$ of space.

Z-String. A more compact frequency representation based upon the packet payload's byte distribution is what we term a "Z-String", short for "Zipf String" [46]. As its name implies, when a byte frequency distribution is rank-ordered, it usually produces a Zipf-like distribution (exponentially decreasing frequency values). We rank order the distribution of a suspicious packet from most frequent to least and drop the frequency counts, resulting in a Z-String. A $\mathrm{Z}$-String relies on the relative notion of frequency just by the ordering of the individual byte values, and since it is a string, we can apply the raw matching techniques described above to the ZStrings themselves. Z-Strings are also often smaller than full packets (e.g., 8-bit byte-based packets would be referenced by a 256byte Z-String), and as such the string comparison times are generally shorter than on the raw packets themselves. However, ZStrings still have an $O(n \lg n)$ creation overhead in the size of the alphabet. (See section 4.3 for an example generated Z-String.)

\subsubsection{Binary-modeled $n$-gram alert correlation}

While frequency-modeled 1-gram alerts offer a measure of privacy, 1-gram modeling cannot represent a sequence of characters. For worms and other malicious binary payloads, we may want to capture such sequences, as they may serve as invariants across multiple suspect payloads that can be correlated. As discussed in [45], binary-based modeling produces surprisingly good results and leads to two different possible alert types.

$\mathbf{N}$-gram signature. We can generate a list of n-grams that are found to be suspicious from an originating packet. Such a "signature" is position-independent while capturing specific malicious byte sequences. Given two n-gram signatures, we can simply compute the intersection of the two and threshold the cardinality of the intersected set to determine a similarity score. Such an intersection is linear time in the length of the signatures by using fast set-based data structures; depending on the n-gram size and packet content, this can vary significantly; while most packets are regular and have few n-grams, encrypted traffic, with a very flat byte distribution, can have as many n-grams as the size of the packet itself. In either case, an n-gram signature is a degenerate form of a raw packet; when distributing large n-grams, this is clearly not privacy-preserving, as even a 5-gram can contain a password. In these cases, we need a transformation on the n-gram itself.

Bloom filter n-gram signature. Instead of publishing an n-gram signature, we can instead insert the n-grams into a Bloom filter and publish it. ${ }^{2}$ Since Bloom filters support both insert and verify, set

\footnotetext{
${ }^{2}$ This is not to be confused with Anagram's use of a BF model; here, individual alerts are placed into Bloom filters.
} 
intersections can be done between a (local) "raw" n-gram signature and a published BF n-gram signature, identifying the same n-grams as the previous technique without yielding other, potentially sensitive n-grams. This approach is also linear in time but leverages a BF's space efficiency. Optionally, multiple alerts can be published via a single Bloom filter, treating the $\mathrm{BF}$ as a bag of suspicious n-grams. This enables a multiplicative reduction in the amount of data transmitted and work needed to compute intersections.

Incidentally, correlating two BF n-gram signatures from different sites can be done via a bitwise AND "intersection"; this does not yield actual n-gram content, but may help find commonality between signatures, increasing confidence that the correct common code has been found when correlated against local data. BF intersection can also be used for model comparison, e.g., comparing two Anagram models to see if different sites exhibit similar traffic properties. Experiments on these approaches are outside the scope of this paper and are briefly discussed in section 6 .

\section{RESULTS}

\subsection{Similarity Score}

As discussed in section 3.1, we compute a set of similarity scores for every correlation technique, $0 \leq$ score $\leq 1$, with a higher score implying a more similar pair of alerts.

Raw packets and Z-Strings. For both of these alert types, our basket of string comparisons can be used. For SE, the score is binary: 0 or 1 , where 1 means equality. For LCS and LCSeq, we use the percentage of the common LCS or LCSeq length out of the total length of candidate strings: score $=2 * C /\left(L_{1}+L_{2}\right)$, where $C$ is the length of LCS/LCSeq and $L_{i}$ is the length of string $i$. For ED, larger values imply dissimilarity; we normalize it as score $=1-D /\left(L_{1}+L_{2}\right)$, where $D$ is the computed edit distance and $L_{i}$ the same as LCS/LCSeq.

Frequency distributions. As mentioned before, frequency distributions are compared using Manhattan distance: $M=\sum_{i=1}^{n} \mid x_{i}-$ $y_{i} \mid$, score $=M / 2$.

Raw and BF n-grams. Since we no longer have full packet content, we instead compute the percentage of common n-grams: score $=2 * N_{c} /\left(N_{1}+N_{2}\right)$, where $N_{c}$ is the number of common n-grams and $N_{i}$ the number of suspicious n-grams in alert $i$. If a Bloom filter is used, a count may be kept with it or approximated by $N_{b} / N_{h}$, i.e., the number of bits set divided by the number of hash functions used.

\subsubsection{Testing with real traffic}

To compare the approaches, we randomly sampled HTTP packets from three sources: clean packets collected from $w w w$ and $w w w 1$ (two heavily-trafficked Columbia CS webservers), and malicious packets collected from a sample of attacks (CodeRed, CodeRed II, WebDAV, Mirela, a phpBB forum attack, and an IIS buffer overflow (MS03-022) exploit). These packets were paired off in three sets: 10,000 "good-vs-good" pairs from 100 packets of $w w w$ and $w w w 1$ traffic each, 1,540 "bad-vs-bad" pairs formed in the cross-product of the 56 packet malicious dataset, and 5,600 "good-vs-bad" pairs of $w w w 1$ and malicious packets. Similarity scores were generated for all of the resulting pairs with all techniques, except SE, which is too brittle to produce meaningful comparisons, and the n-gram analyses, which cannot be compared over an entire packet.

Figure 1 visualizes a small random subset ( 80 pairs) of the scores generated from the "good-vs-good" source. As figure 1 shows, the performance plots of the methods appear similar, although their centers and scale values differ as the scores are not normalized between the correlation methods. On raw payloads, LCSeq and
ED bear very similar results, while comparisons on Z-Strings yield "flatter" results, as less information is compared.

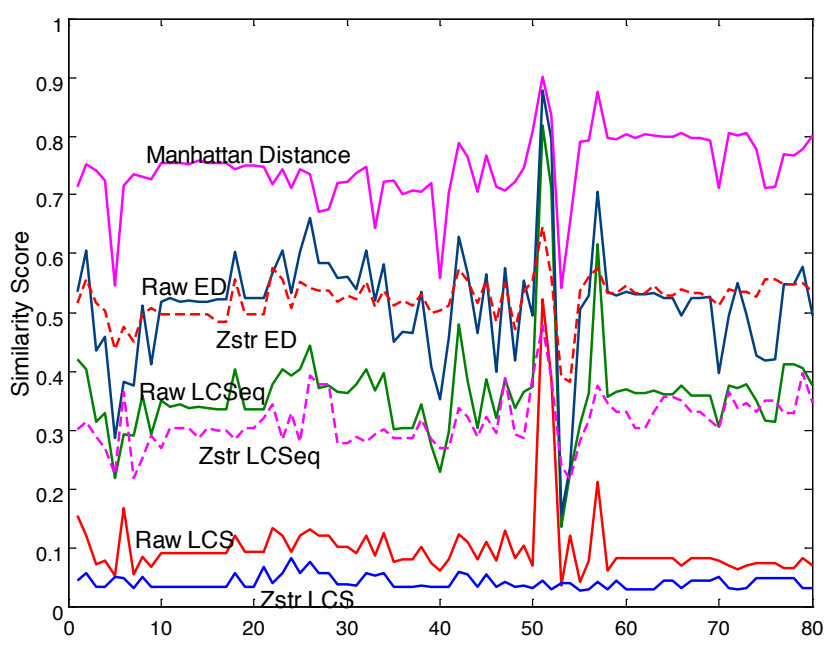

Figure 1: Similarity score comparison of 80 random pairs of "good-vs-good" alerts.

As a more complete experiment, normalized scores were generated and compared for all of the pairs formed amongst the three datasets. To normalize the scores for a comparison, we first compute similarity score vectors $V_{A}, V_{B}$ for the same data over two techniques $A$ and $B$. The center of the two vectors are then aligned by shifting the median of $V_{A}$ to match $V_{B}$. Finally, $V_{A}$ 's range is scaled proportionally so that its min and max values match $V_{B}$ 's. This normalization allows us to compute the Manhattan Distance of the two vectors, distance $=\sum_{i=1}^{n}\left|V_{A_{i}}-V_{B_{i}}\right|$; smaller values imply greater similarity between the two methods. Note that these scores are relative and dependent on the data used; the normalized results are only useful for comparing against a baseline, not as a source of absolute values or across datasets. These pairs were tested with each technique, and the resulting scores were normalized against and compared to the LCSeq score over raw packets. Table 1 shows the computed results.

\begin{tabular}{|c|r|r||r||r|r|r|}
\hline Type & $\begin{array}{r}\text { Raw- } \\
\text { LCS }\end{array}$ & $\begin{array}{r}\text { Raw- } \\
\text { ED }\end{array}$ & MD & $\begin{array}{r}\text { ZStr- } \\
\text { LCS }\end{array}$ & $\begin{array}{r}\text { ZStr- } \\
\text { LCSeq }\end{array}$ & $\begin{array}{r}\text { ZStr- } \\
\text { ED }\end{array}$ \\
\hline \hline G-G & .0948 & .0336 & .0669 & .2079 & .0794 & .0667 \\
\hline B-B & .0508 & .0441 & .0653 & .0399 & .0263 & .0669 \\
\hline G-B & .0251 & .0241 & .0110 & .0310 & .0191 & .0233 \\
\hline
\end{tabular}

Table 1: Manhattan distance from Raw-LCSeq; lower is better.

Averaged over the three scores, Raw-ED is, unsurprisingly, closest to Raw-LCSeq. When privacy-preserving methods are considered, Manhattan distance performs the best overall, and particularly well for good-vs-bad comparison. All of the privacy-preserving methods are close when correlating pairs with attack traffic; we conjecture that significantly different byte distributions enable effective comparison even when some information is lost via privacypreservation.

\subsection{Cross-Domain Alert Correlation}

Next, we compare the techniques by examining their actual performance in identifying true alerts from false positives. Ideally, all false alerts are eliminated by a small similarity score (i.e. the site that produced the alert was the only site that saw this suspicious packet) while true alerts are identified with high similarity scores (i.e. the attack has been launched against more than one site). 
padding "noise". Results with different n-gram sizes and another CRII packet are presented in an appendix in the extended version of this paper on our website [31].

* / def*ult.ida?XXXX *XXXX\%u $9090 \%$ u $6858 \%$ ucbd3\% u $7801 \%$ u $9090 \%$ u $6858 \%$ ucbd $3 \%$ u $7801 \%$ u $9090 \%$ u $6858 \% u$ cbd3\%u $7801 \% u 9090 \%$ u $9090 \%$ u $8190 \%$ u 00 c $3 \% u 0003 \% u 8$ b00\%u531b\%u53ff\%u0078\%u0000\%u00=a HT*: 3379

Figure 6: Generated 5-gram signature from the CRII packet; only the first 172 bytes are shown for brevity.

\subsection{Measuring Privacy Gain}

As discussed earlier, we can use a probabilistic model as a firstorder approximation to measure the relative privacy of each privacypreserving technique.

Frequency-based approaches. Recovery of the original text from its privacy-preserving encoding can be modeled as follows: given a frequency distribution $f=\left\{\left(b_{0}, \pi_{0}\right),\left(b_{1}, \pi_{1}\right), \ldots,\left(b_{n-1}\right.\right.$, $\left.\left.\pi_{n-1}\right)\right\}$, where $n$ is the size of the alphabet, $b_{i}$ is the byte value with probability $\pi_{i}$, and a target length $l$, we construct the content of a packet $p=\left\{b_{0} b_{0} \ldots b_{0} b_{1} b_{1} \ldots b_{1} \cdots b_{n-1} b_{n-1} \ldots b_{n-1}\right\}$, with $\pi_{0} l$ copies of $b_{0}, \pi_{1} l$ copies of $b_{1}$, and so on.

We can now characterize the recovery likelihood $R=1$ / $(l ! /((\pi 0 l) !(\pi 1 l) ! \ldots(\pi n-1 l) !))$, where the denominator is simply the count of all permutations of $p$. This is an effective estimate of the privacy of the frequency distribution, as it represents the likelihood an attacker will be able to correctly guess the true content of the original packets. This number is, additionally, vanishingly small. For the frequency distribution of the CRII packet shown in figure $4, R \approx 1 / 2^{8208}$, well beyond the scope of feasibility, despite the packet's regularity thanks to the large padded section.

The value of $R$ for a Z-String is orders-of-magnitude smaller; not only do permutations of a packet $p_{i}$ have to be computed, there are many such packets; since no frequency information is stored, one must guess the frequencies for each of the bytes $b_{i}$ in the packet. In short, effectively guessing the correct base packet $p_{i}$ and its correct permutation is intractable.

$\mathbf{N}$-grams. We can consider the privacy of both a raw collection of $n$-grams and a corresponding Bloom filter encoding. The raw collection is not very privacy-preserving; not only can a byte sequence contain valuable information (e.g., an entire password), significant n-gram collections enable reassembly of much of the original packet, even without position information. Given a 5-gram $\left\{b_{1} b_{2} b_{3} b_{4} b_{5}\right\}$ from packet $p$, one can search for the 5-gram

$\left\{b_{2} b_{3} b_{4} b_{5} b_{x}\right\}$ in the collection, where $b_{x}$ is any byte; if found, one can reasonably assume the presence of the 6-gram $\left\{b_{1} \ldots b_{5} b_{x}\right\}$ in packet $p$; since $256^{4}$, the number of "common" 4-grams contained in both two 5-grams, is much greater than a packet's size, it is highly unlikely that the common 4-gram happens to be a coincidence. Combined with the fact that high-scoring alerts can contain nearly as many n-grams as the original packet's size, this is impractical from a privacy perspective.

Instead, as previously proposed, we insert the collection of ngrams into a Bloom filter before publishing it. The size of the Bloom filter need not be much more than the number of n-grams; we can pick a size, say $2^{12}$ bits, which is more than twice the size of any individual packet and not prone to significant false positives. (This Bloom filter still takes substantially less memory than the ngram collection itself.) Given such an encoding, the only practical way of recovering the data is to brute-force verify every possible $\mathrm{n}$-gram against the Bloom filter. For example, if we know that only 5 -grams are contained in the Bloom filter, there are $256^{5}$ possi- ble n-grams. Not only is testing all such n-grams computationally infeasible, a brute-force attempt is likely to generate many, many false positives, since there are $256^{5} / 2^{12}$ possible n-grams for each set bit in the Bloom filter (assuming one hash function ${ }^{5}$ ); the recovery likelihood $R=\left(2^{12} / 256^{5}\right)^{m}$, where $m$ is the number of $\mathrm{n}$-grams recovered, is again vanishingly small. This number grows even smaller if multiple n-gram sizes are embedded in the same Bloom filter.

Interestingly, despite the number of possible n-grams for each bit of the Bloom filter, correlating such filters is not prone to significant misclassification. We can characterize the "unlucky coincidence" rate $=\left(\frac{256^{5} / 2^{12}}{256^{5}}\right)^{m}$, that is, the likelihood that we happen to incorrectly verify $m$ possible n-grams, each represented by a particular bit $b_{i}$, out of all possible n-grams. This simplifies to $\left(\frac{1}{2^{12}}\right)^{m}$, which rapidly grows smaller with increasing $m$. In our experiments, we found that a similarity score threshold of 0.1 produced good results; combined with the fact that the average number of n-grams in a false positive alert is approximately 55 , the probability of miscorrelating a Bloom filter alert due to 5 unlucky coincidences is $\left(\frac{1}{2^{12}}\right)^{5}$-not a major concern. In short, testing multiple n-grams eliminates coincidences very rapidly. Sizing the Bloom filter appropriately to avoid saturation is a far more important issue.

Given the effectiveness of n-gram analysis, combined with its strong privacy guarantees and compact size, we believe there is great promise for this form of payload-based correlation.

\section{RELATED WORK}

We discuss selected related work from a number of different network security and intrusion-detection areas, and encourage readers to see the related work sections of $[46,44,45]$ for a full discussion on network anomaly detection, frequency and n-gram analysis. To the best of our knowledge, there does not exist a collection of techniques nor an evaluation similar in scope to this paper.

Distributed intrusion detection. Distributed intrusion detection has been researched for over 10 years; most research, e.g., [35, $32,23]$ has focused on distribution within an enclave, although recent work [2, 17, 49] has looked at Internet-scale correlation and detection. These approaches primarily focus on packet header information and none of them are privacy-preserving; some use endto-end encryption, but this does not alleviate the notion of sensitive data exchange. DShield [40] is the most active volunteer-based DIDS project on the Internet that we are aware of, focusing on "top 10"-style reports and blacklists. DOMINO [49] organizes a decentralized, heterogeneous collection of NIDS sensors; the paper measured, using DShield alert logs, the notion of information gain-and concluded that 40-60 sites enables building summaries and/or blacklists with high degrees of confidence. An interesting application of this approach would be to measure confidence when payloads are involved.

Signature generation and exchange. Another approach is to exchange only "known bad" or exploit-specific signatures. Classic payload-based work in this field includes Earlybird [34], Honeycomb [21], and Autograph [18]. These approaches generally implement string-style payload comparison algorithms, including LCS, LCSeq, and Rabin fingerprints, and can be considered alongside the baseline techniques discussed here. Polygraph [30] explicitly addresses the notion of polymorphic worms using LCSeqlike techniques; the authors show that even polymorphic attacks must contain invariant substrings, a fact we leverage. FLIPS [28] pairs PAYL with an Instruction Set Randomization infrastructure for zero-day worm signature generation. PADS [39], or "Position-

\footnotetext{
${ }^{5}$ Additional hash functions do not affect our analysis.
} 
Aware Distribution Signatures", seek to blend frequency distributions and packet signature positioning.

More recently, work has focused on building semantic-aware or vulnerability-based signatures to handle multiple (or polymorphic) attacks for the same exploit. Kruegel et. al. [22] use structural analysis of binary code and generate control-flow graphs to catch worm mutations. Shield [42] provides vulnerability-specific but exploit-generic filters based on predefined protocol-based policies. Vigilante [8] introduces the notion of vulnerability-specific selfcertifying alerts that focus on filtering undesirable execution control, code execution, or function arguments, and can be exchanged via P2P systems. VSEF [29] builds execution-based filters that filter out vulnerable processor instruction-based traces. COVERS [24] analyzes attack-triggered memory errors on a host and develops structural memory signatures. Nemean [50] uses session-layer and application-protocol semantics to reduce false positives. Some of these signatures and filter descriptions may be exchangeable using our techniques.

Privacy-preserving collaboration. Lincoln et al. [25] suggest hash-based sanitization of several header fields, enabling equality matching (e.g., identifying the same source IP) while removing other features, including payloads; instead, our techniques keep (and analyze) these payloads. Kissner [19] describes the notion of privacy-preserving set operations using cryptographic techniques, achieving stronger privacy guarantees. While it is restricted to set union, intersection, etc., it may potentially support n-gram analysis. Privacy-Preserving Friends Troubleshooting Network [16] extends earlier work on PeerPressure [43] — collaborative software configuration diagnosis - with a privacy-preserving architecture utilizing a "friend"-based neighbor approach, including the use of secure multiparty computation to vote on configuration outliers and homomorphic encryption to protect privacy. Xu [47] introduces the notion of "concept hierarchies" to abstract low-level concepts, along with the use of entropy, to balance the sanitization and information gain of alerts; a similar use of entropy may also be applicable here. The JAM project in our research group [38] was an early inspiration for this work; it originally looked at abstracting and comparing models of data for bank fraud to enable competing financial institutions to collaborate in catching criminals without releasing sensitive information. This work, on the other hand, focuses on the validation of byte content detected at multiple sites as indicators of common attack information; the JAM work more closely resembles BF model intersection, which we briefly discuss in section 6 .

Privacy-preserving databases and data mining. There is a tremendous volume of work on various aspects of data mining and databases, e.g. [1]; these primarily work offline, and assume secure querying, perturbation, and aggregate computation of values amongst one or two databases, and do not generally scale to the run-time correlation described here.

Bloom filters. Bloom filters have been used for secure indexing and data exchange [4, 3, 14]; most focus on two-party interaction and precisely-defined entities. [12,6] use Bloom filters for hardware-based packet inspection and classification.

Secure multiparty computation (SMC) [48] is a theoretically attractive way to accomplish privacy-preservation, e.g., intersection can be fashioned as a secure computation problem. Du et al. [13] briefly reviews the possibility of sharing intrusion detection information via SMC; however, algorithmic cost remains a concern [25], especially with large alert streams.

\section{FUTURE WORK}

Wide-scale deployment, cross-site evaluation. These techniques are specifically designed to be deployed at many enclaves to increase the correlation power and confidence provided by sensors at different sites with different content flows. Our Worminator distributed collaborative intrusion detection platform [37, 26] focused on slow, stealthy alert scan correlation with COTS IDS sensors. We are integrating the work reported in this paper with a new and substantially different content exchange and sharing network known as DNAD-2 (Distributed Network Anomaly Detection) and seek collaborators to share their respective suspicious content detected by whatever local sensors may be available to them.

Polymorphic/obfuscated worm detection and mimicry attack. As suggested by section 4, n-gram analysis has the potential of detecting polymorphic worms, e.g., [11]. While the problem becomes significantly harder as polymorphic worm engines launch mimicry attacks $[41,20]$ to mask themselves, such attacks are generally site-specific. Intersecting $\mathrm{n}$-gram BFs across sites may provide the opportunity to identify even the few bytes of invariant common "code" that appear anywhere in such attacks. Having more BFs that can correlate these short regions increases the confidence in having found the correct snippets. High-entropy regions, such as those containing polymorphic or obfuscated code, would likely not be correlated.

Privacy-preserving model correlation. Given different site anomaly models, e.g., two Bloom filters that represent anomaly models for Anagram, we can do a bitwise AND of the two Bloom filters to estimate the number of common "good" n-grams, or a bitwise OR of the two Bloom filters to aggregate and update the respective models. Further discussion of this concept is beyond the scope of this paper; see [15,9] for an application of this approach to enhance access control.

\section{CONCLUSION}

We have presented a view of cross-site and cross-domain collaborative security by way of sharing content-based alerts among sites. It is to everyone's benefit to share important information without violating policies that inhibit the disclosure of information. In particular, content-based alerts generated by locally-trained payload anomaly detectors reveals an opportunity to detect the early onset of zero-day worm or targeted attacks. We presented a comparative evaluation of alternative correlation strategies and accuracy measures using test data sets with known worm exploits, and included a proposed estimate of the "privacy gain" each method affords. This is important in approaching the problem analytically in order to help break down barriers to collaboration. We find that cross-site and cross-domain privacy-preserving "suspect payload" alert sharing is feasible and useful as revealed in the analysis of Bloom filterexchanged alerts encoding suspect anomalous n-grams.

The techniques hold promise for other purposes as well. For example, sites may exchange their respective anomaly detection models to measure their respective "content flow diversity", enabling estimation of the relative value of different anomaly alerts generated by different sites. More similar sites may have a higher chance of detecting common exploits. Finally, privacy-preserving content alerts may also be useful for other problems, such as collaborative spam filtering, suspicious content detection for botnet command-and-control data streams, etc.

\section{ACKNOWLEDGMENTS}

We would like to thank Gabriela Cretu, Wei-Jen Li, Vanessa FriasMartinez, Michael Locasto, Angelos Stavrou, and Angelos Keromytis for their feedback and collaboration on our design. We would also like to thank Panagiotis Manolios and Peter Dillinger for their suggestions in Bloom filter design. This work was partially sponsored under a grant with the Army Research Office, No. DA W911NF-04-1-0442. 


\section{REFERENCES}

[1] R. Agrawal and R. Srikant. Privacy-Preserving Data Mining. In ACM SIGMOD, 2000.

[2] K. G. Anagnostakis, M. B. Greenwald, S. Ioannidis, A. D. Keromytis, and D. Li. A Cooperative Immunization System for an Untrusting Internet. In IEEE International Conference on Networks, 2003.

[3] M. Bawa, R. J. Bayardo Jr., and R. Agrawal. Privacy-Preserving Indexing of Documents on the Network. In $V L D B, 2003$.

[4] S. M. Bellovin and W. R. Cheswick. Privacy-Enhanced Searches Using Encrypted Bloom Filters, 2004.

[5] B. H. Bloom. Space/time trade-offs in Hash Coding with Allowable Errors. Communications of the ACM, 13(7):422-426, 1970.

[6] F. Chang, W.-c. Feng, and K. Li. Approximate Caches for Packet Classification. In IEEE INFOCOM, 2004.

[7] E. Cooke, F. Jahanian, and D. McPherson. The Zombie Roundup: Understanding, Detecting and Disrupting Botnets. In USENIX SRUTI Workshop, Cambridge, MA, 2005.

[8] M. Costa, J. Crowcroft, M. Castro, A. Rowstron, L. Zhou, L. Zhang, and P. Barham. Vigilante: End-to-End Containment of Internet Worms. In ACM SOSP, 2005.

[9] G. Cretu, J. J. Parekh, K. Wang, and S. J. Stolfo. Intrusion and Anomaly Detection Model Exchange for Mobile Ad-Hoc Networks. In IEEE Consumer Communications and Networking Conference, Las Vegas, NV, 2006.

[10] D. Dagon, C. Zou, and W. Lee. Modeling Botnet Propagation Using Time Zones. In Network and Distributed System Security Symposium (NDSS), San Diego, CA, 2006.

[11] T. Detristan, T. Ulenspiegel, Y. Malcom, and M. von Underduk. Polymorphic Shellcode Engine Using Spectrum Analysis, 2003.

[12] S. Dharmapurikar, P. Krishnamurthy, T. Sproull, and J. Lockwood. Deep Packet Inspection using Parallel Bloom Filters. In IEEE Symposium on High Performance Interconnects (HOTI), 2003.

[13] W. Du and M. Atallah. Secure Multi-Party Computation Problems and Their Applications: A Review and Open Problems. In New Security Paradigms Workshop, 2001.

[14] L. Fan, P. Cao, J. Almeida, and A. Broder. Summary Cache: A Scalable Wide-Area Web Cache Sharing Protocol. In ACM SIGCOMM, 1998.

[15] V. Frias-Martinez and S. J. Stolfo. BARTER: Profile Model Exchange for Behavior-based Access Control. Technical report, Columbia University, 2006. Submitted to conference.

[16] Q. Huang, H. J. Wang, and N. Borisov. Privacy-Preserving Friends Troubleshooting Network. In NDSS, San Diego, CA, 2005.

[17] R. Janakiraman, M. Waldvogel, and Q. Zhang. Indra: A peer-to-peer approach to network intrusion detection and prevention. In WETICE, 2003.

[18] H.-A. Kim and B. Karp. Autograph: Toward Automated, Distributed Worm Signature Detection. In USENIX Security Symposium, San Diego, CA, 2004.

[19] L. Kissner and D. Song. Privacy-Preserving Set Operations. In CRYPTO, 2005.

[20] O. Kolesnikov, D. Dagon, and W. Lee. Advanced Polymorphic Worms: Evading IDS by Blending in with Normal Traffic, 2006

[21] C. Kreibich and J. Crowcroft. Honeycomb - Creating Intrusion Detection Signatures Using Honeypots. In ACM Workshop on Hot Topics in Networks, Boston, MA, 2003.

[22] C. Kruegel, E. Kirda, D. Mutz, W. Robertson, and G. Vigna. Polymorphic Worm Detection Using Structural Information of Executables. In Symposium on Recent Advances in Intrusion Detection, Seattle, WA, 2005.

[23] C. Kruegel, T. Toth, and C. Kerer. Decentralized Event Correlation for Intrusion Detection. In International Conference on Information Security and Cryptology, 2002.

[24] Z. Liang and R. Sekar. Fast and Automated Generation of Attack Signatures: A Basis for Building Self-Protecing Servers. In ACM Conference on Computer and Communications Security, Alexandria, VA, 2005.

[25] P. Lincoln, P. Porras, and V. Shmatikov. Privacy-Preserving Sharing and Correlation of Security Alerts. In USENIX Security, 2004

[26] M. E. Locasto, J. J. Parekh, A. D. Keromytis, and S. J. Stolfo.
Towards Collaborative Security and P2P Intrusion Detection. In IEEE Information Assurance Workshop, West Point, NY, 2005.

[27] M. E. Locasto, S. Sidiroglou, and A. D. Keromytis. Software Self-Healing Using Collaborative Application Communities. In Internet Society (ISOC) Symposium on Network and Distributed Systems Security, pages 95-106, San Diego, CA, 2006.

[28] M. E. Locasto, K. Wang, A. D. Keromytis, and S. J. Stolfo. FLIPS: Hybrid Adaptive Intrusion Prevention. In Symposium on Recent Advances in Intrusion Detection, Seattle, WA, 2005.

[29] J. Newsome, D. Brumley, and D. Song. Vulnerability-Specific Execution Filtering for Exploit Prevention on Commodity Software. In Network and Distributed Security Symposium (NDSS), San Diego, CA, 2006.

[30] J. Newsome, B. Karp, and D. Song. Polygraph: Automatically Generating Signatures for Polymorphic Worms. In IEEE Security and Privacy, Oakland, CA, 2005.

[31] J. J. Parekh, K. Wang, and S. J. Stolfo. Privacy-Preserving Payload-Based Correlation for Accurate Malicious Traffic Detection. Technical report, 2006. http://mice.cs.columbia.edu/ get Techreport . php?techreport ID $=409$.

[32] P. Porras and P. G. Neumann. EMERALD: Event Monitoring Enabling Responses to Anomalous Live Disturbances. In National Information Systems Security Conference, 1997.

[33] H. Project and R. Alliance. Know your Enemy: Tracking Botnets, 3/13/05 2005. http: //www. honeynet. org/papers/bots/.

[34] S. Singh, C. Estan, G. Varghese, and S. Savage. Automated Worm Fingerprinting. In 6th Symposium on Operating Systems Design and Implementation (OSDI '04), San Francisco, CA, 2004.

[35] S. Staniford-Chen, S. Cheung, R. Crawford, and M. Dilger. GrIDS A Graph Based Intrusion Detection System for Large Networks. In National Information Computer Security Conference, Baltimore, MD, 1996.

[36] S. Staniford-Chen, V. Paxson, and N. Weaver. How to Own the Internet in Your Spare Time. In USENIX Security, 2002.

[37] S. J. Stolfo. Worm and Attack Early Warning: Piercing Stealthy Reconnaissance. IEEE Security and Privacy, 2004.

[38] S. J. Stolfo, A. L. Prodromidis, S. Tselepis, W. Lee, D. W. Fan, and P. Chan. JAM: Java Agents for Meta-Learning over Distributed Databases. In International Conference on Knowledge Discovery and Data Mining, Newport Beach, CA, 1997.

[39] Y. Tang and S. Chen. Defending Against Internet Worms: A Signature-Based Approach. In IEEE Infocom, Miami, FL, 2005.

[40] J. Ullrich. DShield home page, 2005. http://www.dshield.org.

[41] D. Wagner and P. Soto. Mimicry Attacks on Host-Based Intrusion Detection Systems. In ACM CCS, 2002.

[42] H. J. Wang, C. Guo, D. R. Simon, and A. Zugenmaier. Shield: Vulnerability-Driven Network Filters for Preventing Known Vulnerability Exploits. In ACM SIGCOMM, 2004.

[43] H. J. Wang, J. C. Platt, Y. Chen, R. Zhang, and Y.-M. Wang. Automatic Misconfiguration Troubleshooting with PeerPressure. In OSDI, San Francisco, 2004.

[44] K. Wang, G. Cretu, and S. J. Stolfo. Anomalous Payload-based Worm Detection and Signature Generation. In Symposium on Recent Advances in Intrusion Detection, Seattle, WA, 2005.

[45] K. Wang, J. J. Parekh, and S. J. Stolfo. Anagram: A Content Anomaly Detector Resistant to Mimicry Attack. In Symposium on Recent Advances in Intrusion Detection, Hamburg, Germany, 2006.

[46] K. Wang and S. J. Stolfo. Anomalous Payload-based Network Intrusion Detection. In Symposium on Recent Advances in Intrusion Detection, Sophia Antipolis, France, 2004

[47] D. Xu and P. Ning. Privacy-Preserving Alert Correlation: A Concept Hierarchy Based Approach. In 21st Annual Computer Security Applications Conference, Tucson, AZ, 2005.

[48] A. C. Yao. Protocols for Secure Computations. In IEEE Symposium on Foundations of Computer Science, 1982.

[49] V. Yegneswaran, P. Barford, and S. Jha. Global Intrusion Detection in the DOMINO Overlay System. In NDSS, 2004.

[50] V. Yegneswaran, J. T. Giffin, P. Barford, and S. Jha. An Architecture for Generating Semantics-Aware Signatures. In USENIX Security Symposium, 2005. 